\title{
Upper Body Lift: Serie de casos en un hospital clínico universitario entre los años 2013-2016. Descripción de la técnica quirúrgica
}

\author{
Stefan Danilla E. ${ }^{1}$, Carlos Domínguez C. ${ }^{1}$, José Tomas Ganz V. ${ }^{2}$, \\ Ekaterina Troncoso O. ${ }^{1}$, Marco Ríos V. ${ }^{1}$, Naomi Yamada T. ${ }^{1}$, Sofía Serra D. ${ }^{1}$, \\ Patricio Andrades C. ${ }^{1}$, Juan Pablo Cisternas V. ${ }^{1}$, Cristian Erazo C. ${ }^{1}$ y Sergio Sepúlveda P. ${ }^{1}$
}

'Departamento de Cirugía Hospital Clínico de la Universidad de Chile, Santiago, Chile. ${ }^{2}$ Hospital Clínico Padre Hurtado.

Recibido el 12 de julio de 2017 y aceptado para publicación el 14 de septiembre de 2017

Correspondencia a: Dr. Stefan Danilla E. drstefandanilla@gmail.com
Upper Body Lift: Cases series from a clinical hospital between years 2013-2016, and description of the surgical technique

Introduction: Obese patients gets beneficial health effects with the massive weight loss, however they develop excessive redundant skin and become physical problems affecting their quality of life. In severe cases, the conventional body contouring techniques are not able to solve this large alterations, being necessary to use more extensive procedures. The Upper Body Lift is a technique that offers integral management of the upper trunk. Objectives: To describe a case series of patients submitted to Upper Body Lift and present the surgical technique. Materials and Methods: Prospective case series of patients with Upper Body Lift between January 2013 and June 2016 in a clinical hospital. Patients with incomplete information or operated in another center were excluded. Descriptive statistics was used. Results: 8 patients were included, age $39.75 \pm 9.37$ years, $6(75 \%)$ women. Prior to the contouring surgery they lose $36.75 \pm 9.11$ $\mathrm{kg}$, reaching a BMI of $25.97 \pm 2.35 \mathrm{~kg} / \mathrm{m}^{2}$. In men the chest region was managed with liposuction and subsequent nipple graft, in women mastopexy without implants was performed in 5 . No major complications was observed, 4 had minor complications during the postoperative period. Discussion: This series presents similar results to those described by other authors. Conclusions: The Upper Body Lift offers comprehensive and successful management in patients with large upper body disturbance.

Key words: weight loss; plastic surgery; upper extremity.

\section{Resumen}

Introducción: En pacientes obesos la pérdida masiva de peso presenta efectos beneficiosos para la salud, sin embargo, suelen cursar con un excedente cutáneo que genera problemas físicos, afectando la calidad de vida. En casos severos las técnicas convencionales de contorno corporal, no permiten dar solución a grandes alteraciones, siendo necesario utilizar procedimientos más extensos. El Upper Body Lift es una técnica que ofrece un manejo integral del tronco superior. Objetivos: Describir una serie de casos de pacientes sometidos a Upper Body Lift y presentar la técnica quirúrgica. Material y Método: Serie de casos prospectiva entre enero del 2013 y junio de 2016, en un hospital clínico universitario. Se excluyó a pacientes con información incompleta u operados en otro centro. Se utilizó estadística descriptiva. Resultados: 8 pacientes manejados mediante Upper Body Lift, con edad 39,75 \pm 9,37 años, $6(75 \%)$ mujeres. Con pérdida de peso de $36,75 \pm 9,11 \mathrm{Kg}$, alcanzando un IMC de $25,97 \pm 2,35 \mathrm{Kg} / \mathrm{m}^{2}$. En hombres la región pectoral se manejó con liposucción y posterior injerto de pezón, en mujeres se realizó mastopexia sin implantes en 5 (83,33\%). No se observó complicación mayor y en 4 casos (50\%) se presentó complicación menor durante posoperatorio. Discusión: Esta serie presenta resultados similares a lo descrito por otros autores. Conclusiones: El Upper Body Lift es un procedimiento que ofrece un manejo integral y con buenos resultados, en pacientes con gran excedente cutáneo en tronco superior.

Palabras clave: pérdida de peso; cirugía plástica; extremidad superior. 


\section{Introducción}

En Chile y el mundo la obesidad se ha consolidado como un problema de salud pública debido a su alta prevalencia, comorbilidades asociadas e impacto negativo en la expectativa de vida ${ }^{1-3}$.

La cirugía bariátrica es un tratamiento seguro y eficaz para el manejo de la obesidad, capaz de cambiar la historia natural de esta enfermedad, logrando bajas de peso significativas y sostenidas en el tiempo, así como también, mejoría de sus comorbilidades asociadas. Por tal motivo, durante los últimos años, se ha visto un aumento sostenido en el número de procedimientos realizados a nivel nacional e internacional ${ }^{4}$.

Si bien, los problemas de salud relacionados con la obesidad mejoran significativamente después de la pérdida de $\mathrm{peso}^{4}$, la capacidad de retracción de la piel no siempre permite compensar la reducción de volumen del cuerpo, dejando deformidades importantes en el contorno corporal.

Pacientes con grandes bajas de peso suelen cursar con excedentes cutáneos significativos y formación de pliegues redundantes, que son causa de problemas físicos y de salud mental que afectan la calidad de vida de los pacientes 5 .

La cirugía de contorno corporal ofrece estrategias reconstructivas que buscan corregir las secuelas cutáneas que se generan producto de la baja masiva de peso con un impacto significativo en la calidad de vida de los pacientes ${ }^{6,7}$. Si bien los procedimientos de tronco inferior, como la abdominoplastía, son los más realizados, aquellos pacientes con grandes pérdidas de peso $(35-45 \mathrm{~kg})$ suelen requerir procedimientos en ambos territorios ${ }^{4,8,9}$.

En tronco superior se observa un excedente cutáneo circunferencial y vertical, asociado a un deslizamiento de los tejidos hacia distal ${ }^{5}$.

En la región torácica lateral se describe la formación de un pliegue producto del excedente dermograso, el cual puede extenderse en grado variable hacia el dorsal (back rolls). En relación a las mamas, las mujeres pueden presentar hipertrofia, ptosis o atrofia, asociado a descenso del surco submamario. En hombres se puede presentar grados variables de ginecomastia o pseudoginecomastia ${ }^{5}$. Los brazos presentan sacos colgantes de excedente dérmico que se extiende desde el codo hasta la axila, pudiendo extenderse hasta la porción lateral de la mama ${ }^{5,10}$.

Existen diversas estrategias reconstructivas que permiten corregir las secuelas de la pérdida de peso en el tronco superior ${ }^{10,11}$. El Upper Body Lift (torsoplastía de hemicuerpo superior) es un procedimiento que considera un manejo integral de todo el tronco superior, destinado a pacientes con secuelas de contorno corporal importantes producto de la baja masiva de peso. Es un procedimiento realizado en un tiempo quirúrgico que combina una reducción mamaria, abdominoplastía reversa, braquioplastía y plastía de axila ${ }^{5}$. Dada la extensión del procedimiento y potenciales complicaciones asociadas, su uso se suele reservar para pacientes con un excedente cutáneo de tronco superior extenso, donde los procedimientos convencionales no permiten dar un resultado satisfactorio ${ }^{5,12}$.

El presente trabajo tiene por objeto describir una serie clínica de pacientes sometidos a Upper Body Lift para el manejo integral del tronco superior en pacientes con baja masiva de peso y describir la técnica quirúrgica.

\section{Materiales y Métodos}

Serie de casos clínicos prospectiva, obtenida de la base de datos digital de pacientes con cirugía de contorno corporal (Body-QoL). Se incluyó a todos los pacientes sometidos al procedimiento Upper Body Lift entre el 01de enero de 2013 y 31 de junio de 2016, por el equipo de cirugía plástica. Se excluyó los procedimientos realizados en otro centro, en más de un tiempo quirúrgico o con antecedentes incompletos.

Se evaluaron variables demográficas, clínicas, técnica quirúrgica y complicaciones. Las variables continuas se describieron con promedio y desviación estándar, las variables categóricas, con frecuencia y porcentajes. Se realizó un análisis estadístico descriptivo, utilizando STATA $13^{\circledR}$.

\section{Técnica quirúrgica: Upper Body Lift}

\section{Selección de pacientes}

Pacientes seleccionados para este procedimiento presentaban un excedente cutáneo circunferencial y vertical en el tronco superior, presentando descenso marcado del pliegue inframamario lateral, ptosis mamaria y un excedente cutáneo redundante en brazos. Como concepto, el Upper Body Lift implica: 1) Liposucción de los colgajos a resecar; 2) Tratamiento de la mama (con reducción, pexia con aumento o tratamiento de la ginecomastía en hombres); 3) Abdominoplastía reversa; 4) Braquioplastía y 5) Axilo-torsoplastía.

Son candidatos a esta cirugía, pacientes con edad menor a 60 años, comorbilidades estabilizadas, hábito tabáquico suspendido e índice de masa corporal (IMC) $<30 \mathrm{~kg} / \mathrm{m}^{2}$. 


\section{ARTÍCULO ORIGINAL}

\section{Marcación y fotografía preoperatoria}

Fotografía clínica de cuerpo completo con tomas cada $45^{\circ}$ hasta completar todo el perímetro del cuerpo. Realizando esto con brazos en posición anatómica, abducidos y flectados. Se registran las mismas secuencias en el posoperatorio a los 3, 6 y 12 meses.

Respecto a la marcación, en brazos se utiliza la técnica del doble elipse descrita por Al Aly tanto para hombres y mujeres. Paciente con los brazos en flexión de $90^{\circ}$ hacia arriba, se marca la línea media por el borde inferior del brazo, desde el codo hasta la axila; sobre el cual se realiza pinch test, que incluye todo el excedente dermograso ubicado sobre la fascia, esto permite conformar la "elipse externa" (Figura 1. Línea roja). La "elipse interna" (Figura 1. Línea azul), corresponde al tejido a resecar, se diseña descontando al trazado de la elipse externa, el espesor del tejido celular subcutáneo (se recomienda utilizar un caliper a cirujanos con

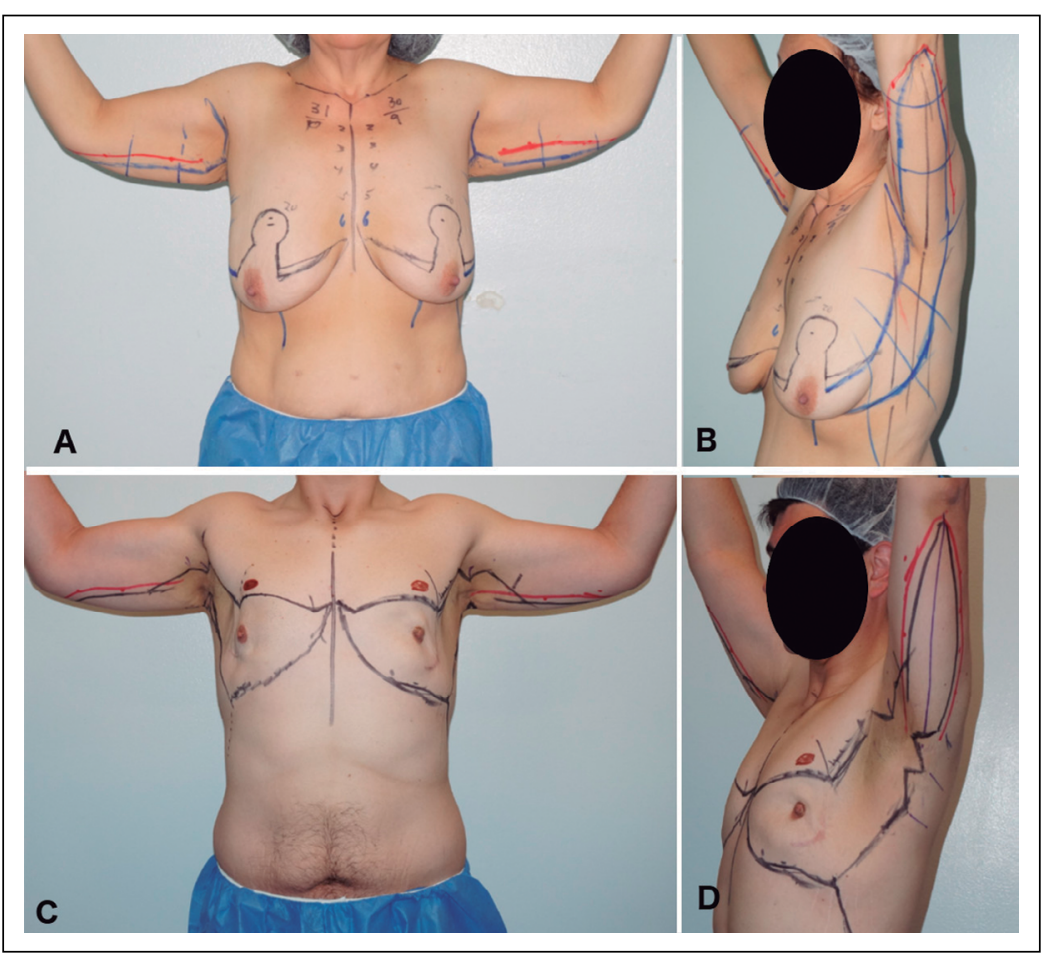

Figura 1. Marcación preoperatoria. A: Paciente sexo femenino, vista frontal, con brazos abducidos y codos flexionados. Se observa marcación de doble elipse en borde inferior de brazos y mastopexia con pedículo superior. B: Paciente sexo femenino, vista lateral, con brazos extendidos. Se observa patrón de resección en brazo, axila y región mamaria lateral (línea azul). C: Paciente masculino, vista frontal con brazos abducidos y codos flexionados. Se observa marcación de doble elipse en borde inferior de brazo, abdominoplastía reversa diseñada en borde inferior de músculo pectoral y marcación de implante de areola. D: Paciente masculino, vista lateral, brazos extendidos. Se observa patrón de resección cutánea en borde posterior de brazo, axila y región torácica lateral (línea azul). poca experiencia y el pinch test solo a cirujanos experimentados).

En la región torácica lateral se define excedente cutáneo a resecar mediante pinch test. Procurando ocultar las cicatrices en el reborde del músculo pectoral mayor en el caso de hombres y en el surco mamario en mujeres. A nivel axilar se completa marcación con el diseño de $\mathrm{Z}$ plastía para evitar contractura en pliegue axilar.

Para la marcación de la mama en mujeres, se diseña mastopexia de pedículo superior, con resección variable de tejido, no obstante, el cirujano puede utilizar la técnica que estime conveniente. Dada la elongación y descenso del surco mamario, este se debe reposicionar a su ubicación habitual a nivel del $6^{\circ}$ arco costal, utilizando una técnica de abdominoplastía reversa. Es importante también destacar que la tracción de tejidos hacia lateral producirá una lateralización del complejo areola pezón, por lo que el cirujano debe "descontar" esta medida a su diseño de marcación de reducción mamaria/mastopexia.

En hombres se utiliza como referencia de marcación el borde inferior del músculo pectoral mayor para diseño de patrón de resección cutánea y se define nueva ubicación del pezón lateral a la línea medio clavicular en una línea oblicua desde la escotadura esternal hasta el borde inferolateral del pectoral.

\section{Técnica quirúrgica}

Tanto vías venosas como monitores de saturación y presión arterial deben ser instalados en los miembros inferiores ya que el cirujano necesitará incluir en el campo ambos miembros superiores.

La posición del paciente en la mesa operatoria dependerá del diseño preoperatorio. De ser necesario una resección torácica lateral hacia posterior (back roll), se inicia el procedimiento en decúbito dorsal para realizar la resección posterior y luego se supina al paciente. De no ser necesario intervenir la dorsal el paciente se posiciona en decúbito dorsal con ambos brazos abducidos incluidos en el campo operatorio.

La cirugía parte con la infiltración de solución vasoconstrictora (epinefrina 1:1.000.000) en toda la zona a resecar de brazos, axila y cara lateral de la mama. Luego, en dichas zonas, se realiza liposucción extensa hasta dejar solo la piel, utilizando cánulas de $4 \mathrm{~mm}$ o $5 \mathrm{~mm}$. Esta maniobra puede parecer fútil, ya que se está lipoaspirando un área que posteriormente será resecada, sin embargo, esta maniobra es clave para evitar complicaciones nerviosas y linfáticas de la cirugía. Una vez realizada la liposucción, se calibra la línea de corte con pinzas 
de Pozzi o Backhouse en toda su extensión y luego se procede al corte. Luego, se reseca la piel en plano subdérmico, preservando todas las estructuras neurovasculares subyacentes del tejido estromal subcutáneo (previamente desgrasado). Una vez resecado el excedente cutáneo, se realiza la sutura inmediata de piel en tres planos, utilizando sutura barbada de lenta reabsorción $2 / 0$ y $3 / 0$ en plano subcutáneo y dermis profunda respectivamente, asociado al uso Dermabond ${ }^{\mathrm{TM}}$ Prineo $^{\mathrm{TM}}$. No se utiliza drenaje.

Posteriormente, se realiza mastopexia a pedículo superior con o sin implante dependiendo de la arquitectura mamaria y de las expectativas de la paciente. Para el reanclaje del surco y abdominoplastía reversa, se elevan los colgajos en plano suprafascial y luego se ancla el surco nativo a la $6^{\mathrm{a}}$ costilla con Ethibond 1. El excedente cutáneo puede resecarse o utilizarse para aumentar las mamas según la técnica descrita por Rubbin.

\section{Principios quirúrgicos claves}

Al igual que en todos los pacientes con baja masiva de peso comprende:

- Mínimo decolamiento.

- Liposucción limitada.

- Cerrar los espacios muertos.

- Proteger hipotermia.

- Evitar uso de drenajes.

\section{Manejo posoperatorio}

Todos los pacientes de UBL o LBL corresponden a pacientes de alto riesgo de tromboembolismo, por lo que se recomienda el uso rutinario de sistemas de compresión neumática intermitente, trombo profilaxis farmacológica con heparinas de bajo peso molecular y el uso de medias antiembólicas. Las
HBPM se continúan habitualmente por al menos 10 días, hasta que el paciente deambule en forma normal.

Se realiza un manejo analgésico multimodal que permite un adecuado manejo del dolor, evitando náuseas y vómitos.

La sonda Foley utilizada durante la intervención, es retirada tempranamente para estimular la deambulación precoz.

No se realiza control de hemoglobina ni hematocrito, salvo indicación puntual del cirujano tratante.

\section{Resultados}

En el período estudiado, se llevaron a cabo un total de 1.027 cirugías de contorno corporal en 120 pacientes con pérdida masiva de peso. De este grupo de 120 pacientes, se realizaron $8(6,67 \%)$ Upper Body Lift, según la técnica anteriormente expuesta. De ellos $6(75 \%)$ casos fueron pacientes de sexo femenino, y $2(25 \%)$ pacientes de sexo masculino, con una edad promedio de 39,75 \pm 9,37 años (rango 26-53) (Tabla 1).

Los pacientes presentaron una baja de peso promedio de $36,75 \pm 9,11 \mathrm{~kg}$, alcanzando un IMC de $25,97 \pm 2,35 \mathrm{~kg} / \mathrm{m}^{2}$ previo al Upper Body Lift; esta disminución de peso fue lograda mediante cirugía bariátrica en $5(62,5 \%)$ (Tabla 1$)$.

En el caso de los hombres, en los brazos, se realizó liposucción y posterior braquioplastía, a nivel torácico liposucción de la región pectoral, resección de excedente cutáneo y posterior reimplante de pezón (Figura 2). En las pacientes de sexo femenino el manejo de brazos comprendió liposucción y posterior braquioplastía, a nivel torácico se realizó

Tabla 1. Tabla resumen, serie de casos Upper Body Lift

\begin{tabular}{|cccccclll}
\hline Caso & Sexo & Edad & $\begin{array}{c}\text { Cirugía } \\
\text { bariátrica }\end{array}$ & $\begin{array}{c}\text { Pérdida } \\
\text { de peso }\end{array}$ & $\begin{array}{c}\text { IMC } \\
\text { precirugía }\end{array}$ & Cirugía mamaria & Complicación \\
\hline 1 & F & 33 & No & 30 & 22,15 & Mastopexia con implante & Cicatriz patológica \\
\hline 2 & M & 30 & No & 40 & 27,41 & Mastectomía + injerto CAP & \\
\hline 3 & F & 53 & Sí & 40 & 26,83 & Mastopexia sin implante & \\
\hline 4 & M & 26 & No & 36 & 25,47 & Mastectomía + injerto CAP & Dehiscencia \\
\hline 5 & F & 46 & Sí & 21 & 30,04 & Mastopexia sin implante & Dehiscencia \\
\hline 6 & F & 48 & Sí & 38 & 23,9 & Mastopexia sin implante & Extrusión material de sutura \\
\hline 7 & F & 41 & Sí & 36 & 26,3 & Mastopexia sin implante & & \\
\hline
\end{tabular}

Se detalla en forma específica el procedimiento realizado en la región mamaria, dado que puede variar dependiendo de cada paciente. 


\section{ARTÍ́CULO ORIGINAL}

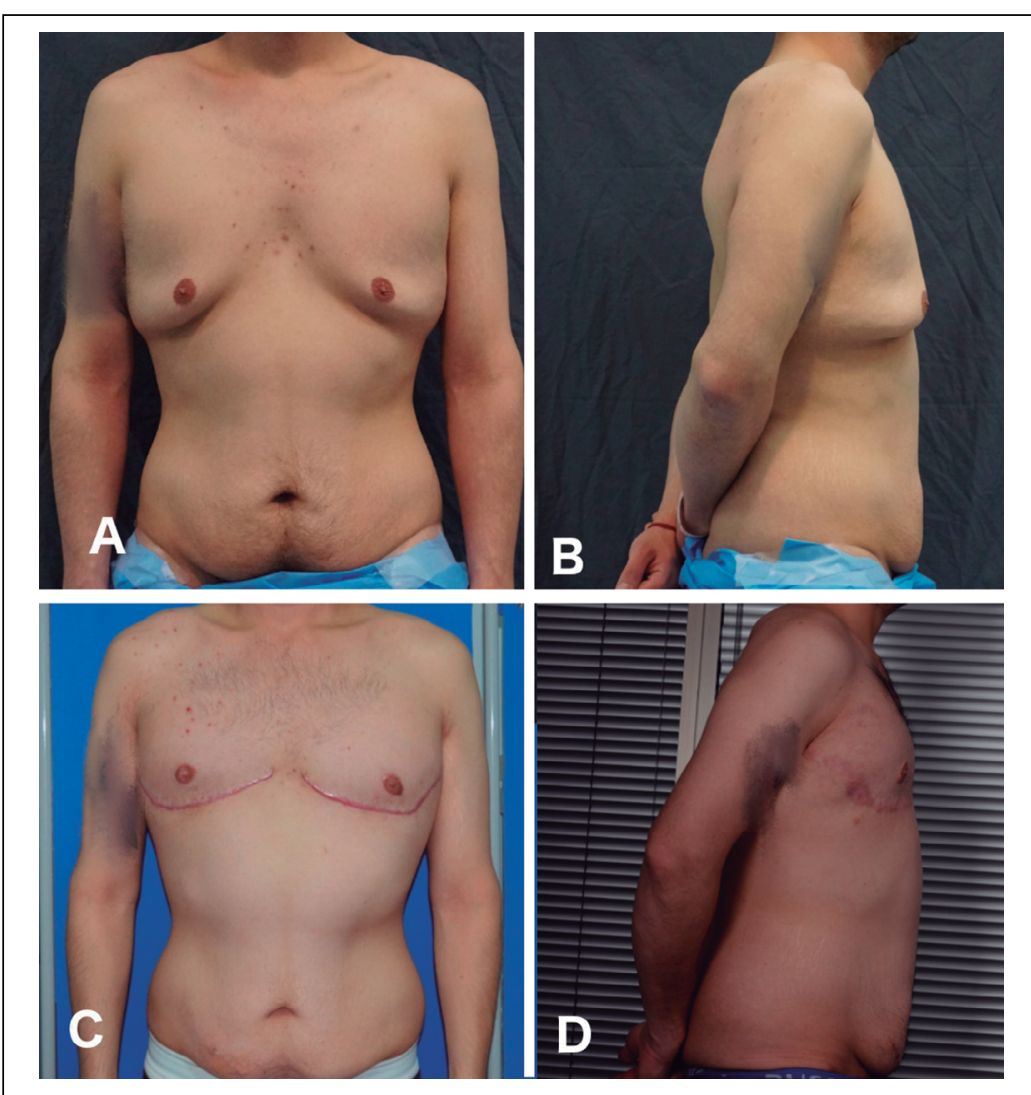

Figura 2. Serie pre y posoperatoria, paciente sexo masculino de 30 años. Braquioplastía, torsoplastía lateral, liposucción resección de excedente cutáneo pectoral con injerto de areola. Se observa cicatriz hipertrófica (c y d).

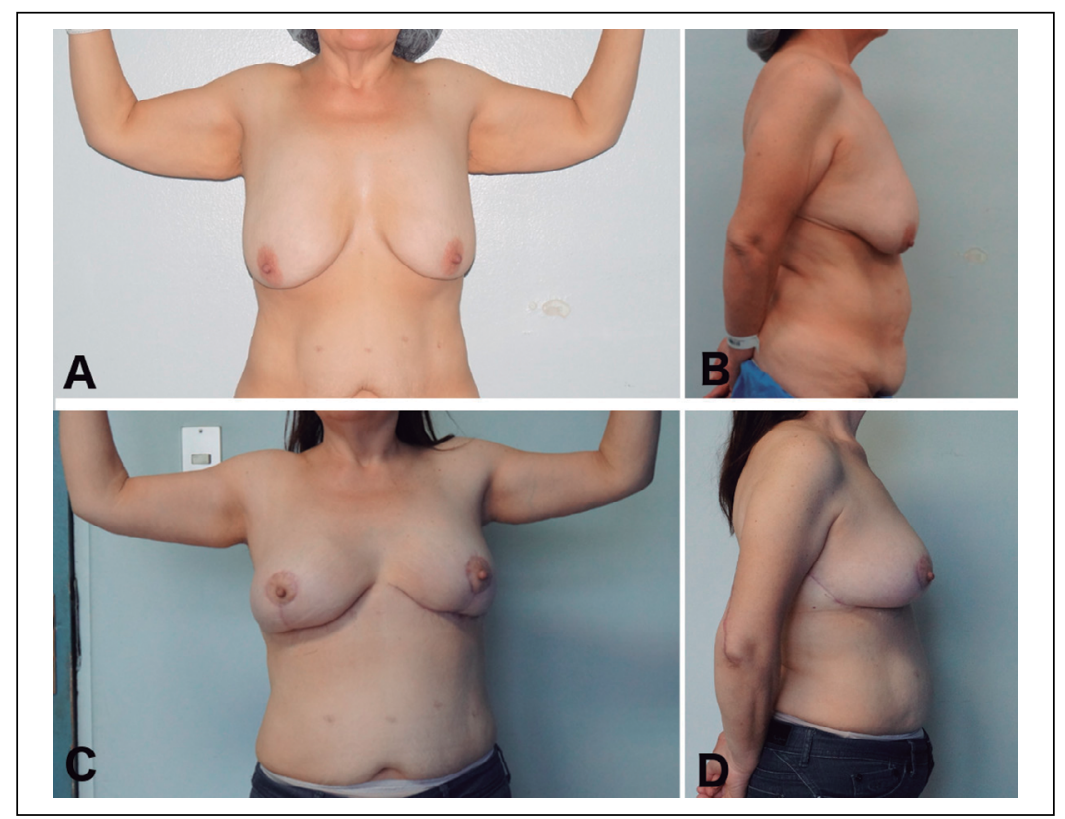

Figura 3. Serie fotográfica pre y posoperatorios, paciente sexo femenino de 41 años. Se realizó braquioplastía, torsoplastía de cara lateral de la mama, mastopexia sin implante con pedículo superior y reposicionamiento de surco mamario. mastopexia sin implantes en 5 paciente, mastopexia con implantes en 1, asociado a reimplante del surco mamario en todos los casos (Figura 3).

El Upper Body Lift se realizó en promedio 1,66 \pm 1,15 años después de la cirugía bariátrica. Presentó un tiempo operatorio promedio de 4,85 $\pm 1,06 \mathrm{~h}^{4-7}$.

Cuatro $(50 \%)$ pacientes se realizaron durante el curso de un año un Lower Body Lift como procedimiento complementario para manejo del tronco inferior.

La serie no registra mortalidad ni complicaciones mayores hospitalarias. Durante el posoperatorio tardío se observó complicaciones menores en 4 (50\%) pacientes: $2(25 \%)$ dehiscencias de sutura, 1 $(12,5 \%)$ queloide y $1(12,5 \%)$ extrusión del material de sutura. Las dehiscencias de heridas se originaron en sitios diferentes; una paciente a nivel axilar siendo manejada con un injerto de piel parcial y el segundo caso a nivel mamario fue manejado mediante reavance de colgajos.

\section{Discusión}

Si bien la pérdida masiva de peso posee indudables efectos positivos en la salud de los pacientes, el exceso de piel es una secuela habitual que puede afectar tanto al tronco superior e inferior, requiriendo grandes resecciones cutáneas. Los pacientes evaluados en esta serie presentaron una pérdida de aproximadamente $36 \mathrm{~kg}$, alcanzando un IMC casi normal al momento de la cirugía de contorno corporal.

El Upper Body Lift es un procedimiento que permitió dar una solución integral y en un solo tiempo quirúrgico a pacientes con secuelas en el tronco superior, producto de la pérdida masiva de peso.

Múltiples series describen procedimientos aislados en diferentes tiempos quirúrgicos para lograr un manejo adecuado del tronco superior ${ }^{10,13,14}$, series clínicas que reporten la experiencia con Upper Body Lift son escasas ${ }^{15,16}$.

Acorde a lo reportado en la literatura, el gran excedente cutáneo circunferencial y vertical, asociado a un pliegue torácico lateral redundante son los principales factores que inclinan la elección de un Upper Body Lift por sobre procedimientos independientes de brazos y mamas 5 .

Al igual que lo referido por Hurwitz ${ }^{17}$ el procedimiento mamario más utilizado fue la mastopexia sin implantes seguido por la mastopexia con implante. Existen, además, reportes del uso de aumentos con tejidos autólogos como colgajos grasos locales ${ }^{16,17}$ o colgajos libres (DIEP, SGAP, muslo interno) ${ }^{18}$. 
El manejo integral del tronco superior en pacientes con baja masiva de peso, mediante la técnica Upper Body Lift es un procedimiento seguro, según lo presentado en nuestra experiencia, la que no presentó mortalidad ni complicaciones mayores. Las complicaciones menores concuerdan con lo reportado por otros grupos en relación al 40-50\% de los pacientes, siendo las relacionadas con la herida operatoria las más frecuentes. En nuestra serie la principal complicación fue la dehiscencia de herida operatoria $(25 \%)$, no observándose desarrollo de seroma, ni infección de herida, que habitualmente son descritas en este tipo de procedimiento ${ }^{13,15,19,20}$. No se presentaron, además, complicaciones funcionales por cicatrices retractiles a nivel axilar, lo cual podría ser atribuido a la incorporación de Z-plastías en el diseño del patrón de resección a nivel axilar.

\section{Conclusiones}

En pacientes con baja masiva de peso, con gran excedente cutáneo en tronco superior, el Upper
Body Lift es un procedimiento que ofrece un manejo integral y con buenos resultados.

\section{Responsabilidades éticas}

Protección de personas y animales. Los autores declaran que para esta investigación no se han realizado experimentos en seres humanos ni en animales.

Confidencialidad de los datos. Los autores declaran que han seguido los protocolos de su centro de trabajo sobre la publicación de datos de pacientes.

Derecho a la privacidad y consentimiento informado. Los autores declaran que en este artículo no aparecen datos de pacientes.

Financiamiento: Sin financiamiento.

Conflictos de interés: Los autores declaran no presentar conflictos de interés.

Agradecimiento: Hospital Clínico de la Universidad de Chile.

\section{Referencias}

1. Vio F, Albala C, Kain J. Nutrition transition in Chile revisited: mid-term evaluation of obesity goals for the period 2000-2010. PHN. 2007;11:1-8.

2. Fountaine K, Redden D, Wang C, Westfall A, Allison D. Years of Life Lost Due to Obesity. JAMA 2003;289:187-93.

3. Encuesta Nacional de Salud 2009-2010 [Internet]. 2011. Available from: http:// www.redsalud.gov.cl/portal/docs/ page/minsalcl/g_home/submenu portada 2011/ens2010.pdf

4. Kitzinger HB, Abayev S, Pittermann A, Karle B, Kubiena H, Bohdjalian A, et al. The Prevalence of Body Contouring Surgery After Gastric Bypass Surgery. Obes Surg. 2011;22:8-12.

5. Soliman S, Rotemberg SC, Pace D, Bark A, Mansur A, Cram A, et al. Upper Body Lift. Clinics in Plastic Surgery 2008;35:107-14.

6. Danilla S, Domínguez C, Cuevas P, Calderón ME, Ríos MA, Andrades P, et al. The Body-QoL( $(\mathbb{B})$ : patient reported outcomes in body contouring surgery patients [corrected]. Aesthetic Plast Surg. Springer US; 2014;38:575-83.

7. Danilla S, Cuevas P, Aedo S, Domínguez C, Jara R, Calderón ME, et al. Introducing the Body-QoL ${ }^{\circledR}$ : A New Patient-Reported Outcome Instrument for Measuring Body Satisfaction-Related Quality of Life in Aesthetic and Postbariatric Body Contouring Patients. Aesthetic Plast Surg. Springer US; 2015 Nov 17:1-13.

8. Kitzinger HB, Cakl T, Wenger R, Hacker S, Aszmann OC, Karle B. Prospective study on complications following a lower body lift after massive weight loss. British Journal of Plastic Surgery. Elsevier Ltd; 2013;66:231-8.

9. Giordano S, Victorzon M, Koskivuo I, Suominen E. Physical discomfort due to redundant skin in post-bariatric surgery patients. British Journal of Plastic Surgery Elsevier Ltd; 2013;66:950-5.

10. Hurwitz DJ, Holland SW. The L Brachioplasty: An Innovative Approach to Correct Excess Tissue of the Upper Arm, Axilla, and Lateral Chest. Plastic and Reconstructive Surgery 2006;117:403-11.
11. Ellison JM, Steffen KJ, Sarwer DB Body Contouring After Bariatric Surgery. de Zwaan M, Mitchell JE, editors. Eur Eat Disorders Rev. 2015;23:479-87.

12. Hasanbegovic E, Sørensen JA. Complications following body contouring surgery after massive weight loss: A meta-analysis. British Journal of Plastic Surgery Elsevier Ltd; 2014;67:295301.

13. Han HH, Lee MC, Kim SH, Lee JH, Ahn ST, Rhie JW. Upper Arm Contouring with Brachioplasty after Massive Weight Loss. Arch Plast Surg. 2014;41:271-6.

14. Rubin JP, Gusenoff JA, Coon D. Dermal Suspension and Parenchymal Reshaping Mastopexy after Massive Weight Loss: Statistical Analysis with Concomitant Procedures from a Prospective Registry. Plastic and Reconstructive Surgery. 2009;123:782-9.

15. Gusenoff JA, Coon D, Rubin JP. Implications of Weight Loss Method in Body Contouring Outcomes. Plastic and Reconstructive Surgery 2009;123:373-6. 


\section{ARTÍCULO ORIGINAL}

16. Huemer GM. Upper Body Reshaping for the Woman with Massive Weight Loss: An Algorithmic Approach. Aesthetic Plast Surg. 2010;34:561-9.

17. Hurwitz DJ, Agha-Mohammadi S. Postbariatric Surgery Breast Reshaping. Annals of Plastic Surgery 2006;56:481-6.
18. Schoeller T, Meirer R, Otto-Schoeller A, Wechselberg G, Piza-Katzer H. Medial Thigh Lift Free Flap for Autologous Breast Augmentation after Bariatric Surgery. Obes Surg. 2002;12:831-4.

19. Almutairi K, Gusenoff JA, Rubin JP. Body Contouring. Plastic and Reconstructive
Surgery. 2016;137:586e-602e.

20. Gusenoff JA, Coon D, Rubin JP. Brachioplasty and Concomitant Procedures after Massive Weight Loss: A Statistical Analysis from a Prospective Registry. Plastic and Reconstructive Surgery 2008;122:595-603. 